\title{
THE STRUCTURE OF PSEUDOCOMPLEMENTED DISTRIBUTIVE LATTICES. II: CONGRUENCE EXTENSION AND AMALGAMATION
}

\author{
G. GRÄTZER AND H. LAKSER( $\left.{ }^{1}\right)$
}

\begin{abstract}
This paper continues the examination of the structure of pseudocomplemented distributive lattices. First, the Congruence Extension Property is proved. This is then applied to examine properties of the equational classes $\mathscr{B}_{n},-1 \leqq n \leqq \omega$, which is a complete list of all the equational classes of pseudocomplemented distributive lattices (see Part I). The standard semigroups (i.e., the semigroup generated by the operators $\boldsymbol{H}, \boldsymbol{S}$, and $\boldsymbol{P}$ ) are described. The Amalgamation Property is shown to hold iff $n \leqq 2$ or $n=\omega$. For $3 \leqq n<\omega, \mathscr{B}_{n}$ does not satisfy the Amalgamation Property; the deviation is measured by a class Amal $\left(\mathscr{B}_{n}\right)\left(\subseteq \mathscr{B}_{n}\right)$. The finite algebras in Amal $\left(\mathscr{B}_{n}\right)$ are determined.
\end{abstract}

0. Introduction. This paper continues the examination of the structure of pseudocomplemented distributive lattices begun in Part I, [8].

Using the description of congruences given in Part I, we verify the Congruence Extension Property in $\S 1$. This, in effect, states that a *-congruence on a subalgebra can be extended to a ${ }^{*}$-congruence on the algebra. This property is applied in $\$ \S 2$ and 3. In $\$ 2$ we determine the "standard semigroups" of the equational classes of pseudocomplemented distributive lattices, which is, roughly speaking, the semigroup generated by the operators $\boldsymbol{H}, \boldsymbol{S}$, and $\boldsymbol{P}$ in the sense of [5]. In $\S 3$ it is shown that the Amalgamation Property holds in $\mathscr{B}_{n}$ (notation of Part I) if and only if $n=-1,0,1,2$, or $\omega$, and that the subalgebra theorem for free products of B. Jónsson [7] holds for exactly the same equational classes. Since the Amalgamation Property fails to hold for $\mathscr{B}_{3}, \mathscr{B}_{4}, \ldots$, we introduce a concept attempting to measure the extent of this failure. This concept is the amalgamation class of $\mathscr{K}$, Amal $(\mathscr{K})$. The Amalgamation Property holds in $\mathscr{K}$ if and only if Amal $(\mathscr{K})=\mathscr{K}$. $\S 4$ contains results on Amal $\left(\mathscr{B}_{n}\right)$ for $2<n<\omega$; in particular, we determine the finite algebras in Amal $\left(\mathscr{B}_{n}\right)$.

1. The Congruence Extension Property. A class $\mathscr{K}$ of algebras is said to satisfy the Congruence Extension Property if, given any algebra $B$ and subalgebra $A$, both in $\mathscr{K}$, and any congruence $\Theta$ on $A$, there is a congruence $\Theta$ on $B$ such that the

Received by the editors July 8, 1970 .

AMS 1970 subject classifications. Primary 06A35; Secondary 08A25, 18A20, 18A30, 18 C05.

Key words and phrases. Distributive lattice, pseudocomplemented, congruence, amalgamation, free product, standard semigroup, amalgamation class.

(1) The research of both authors was supported by the National Research Council of Canada.

Copyright (C) 1971, American Mathematical Society 
restriction of $\Theta$ to $A, \widehat{\Theta}_{A}$, satisfies $\widetilde{\Theta}_{A}=\Theta$. In this section we establish the Congruence Extension Property for the class $\mathscr{B}_{\omega}$ of all pseudocomplemented distributive lattices, where "Congruence" refers to *-congruences, that is, those lattice congruences that also preserve the pseudocomplementation *.

We first recall the characterization of *-congruences established in Part I, [8]. If $L$ is a pseudocomplemented distributive lattice then the set of dense elements $D(L)=\left\{u \in L \mid u^{*}=0\right\}$ is a lattice with 1 and, indeed, is a dual ideal of $L$, and the skeleton $S(L)=\left\{x^{*} \mid x \in L\right\}$ is a Boolean algebra with join operation $a \cup b$ $=\left(a^{*} \wedge b^{*}\right)^{*}$. A congruence pair of $L$ is a pair $\left\langle\Theta_{1}, \Theta_{2}\right\rangle$, where $\Theta_{1}$ is a congruence of $S(L)$ and $\Theta_{2}$ is a congruence of $D(L)$, satisfying

(1) $a \in S(L), u \in D(L), u \geqq a, a \equiv 1\left(\Theta_{1}\right)$ imply $u \equiv 1\left(\Theta_{2}\right)$.

Theorem 1 of Part I established a one-to-one correspondence between *-congruences of $L$ and congruence pairs of $L$; if $\Theta$ is a *-congruence of $L$ then the corresponding congruence pair is $\left\langle\Theta_{S(L)}, \Theta_{D(L)}\right\rangle$, and if $\left\langle\Theta_{1}, \Theta_{2}\right\rangle$ is a congruence pair then the corresponding *-congruence $\Theta$ is determined by requiring that $x \equiv y(\Theta)$ if and only if

(i) $x^{*} \equiv y^{*}\left(\Theta_{1}\right)$ and

(ii) $x \vee u \equiv y \vee u\left(\Theta_{2}\right)$ for all $u \in D(L)$.

As usual, let $(x]$ denote the principal ideal generated by the element $x$.

Lemma 1. Let $K, L$ be distributive lattices with 1 and let $K$ be a $\{1\}$-sublattice of L. Let $\Theta$ be a lattice congruence on $K$ and let $J$ be a dual ideal in $L$ satisfying

(2) $u \in J$, and $(u] \cap K \neq \varnothing$ imply that there is $a v \in(u] \cap K$ such that $v \equiv 1(\Theta)$. Then there is a lattice congruence $\bar{\Theta}$ on L such that the following two conditions are satisfied:

(i) $\bar{\Theta}_{K}=\Theta$;

(ii) $x \equiv 1(\bar{\Theta})$ for all $x \in J$.

Proof. Given elements $a, b \in L$, let $\Theta(a, b)$ denote the smallest congruence on $L$ identifying $a$ with $b$. Let $\Theta[J]$ be the congruence on $L$ determined by $J$; that is, $x \equiv y(\Theta[J])$ if and only if $x \wedge u=y \wedge u$ for some $u \in J$. We define the congruence $\Theta$ on $L$ as

$$
\bar{\Theta}=\Theta[J] \vee \vee(\Theta(a, b) \mid a, b \in K, a \equiv b(\Theta)) .
$$

Clearly condition (ii) holds, and also $\Theta \leqq \bar{\Theta}_{K}$. Thus we need only establish that $\bar{\Theta}_{K} \leqq \Theta$.

Let $x, y \in K, x \not \equiv y(\Theta)$. We show that $x \not \equiv y(\Theta)$. Since $[x] \Theta \neq[y] \Theta$ in the quotient lattice $K / \Theta([x] \Theta,[y] \Theta$ denote the corresponding equivalence classes under $\Theta$ ), they can be separated by a prime ideal $P^{\prime}$ of $K / \Theta$. We may assume without loss of generality that $[x] \Theta \in P^{\prime}$ and $[y] \Theta \in K / \Theta-P^{\prime}$. Let the prime ideal $P$ of $K$ be $\left\{u \in K \mid[u] \Theta \in P^{\prime}\right\}$ and let the prime dual ideal $Q=K-P$, that is, $Q=\left\{u \in K \mid[u] \Theta \notin P^{\prime}\right\}$. Thus $x \in P, y \in Q$. Let $(P]$ be the ideal of $L$ generated by $P$ and let $[Q)$ be the dual ideal of $L$ generated by $Q$. That is, $(P]=\{u \in L \mid u \leqq z$ for some $z \in P\}$ and $[Q)=\{u \in L \mid u \geqq z$ for some $z \in Q\}$. 
We claim that $(P]$ and the dual ideal $[Q) \vee J$ are disjoint. If $u \in(P] \cap([Q) \vee J)$ then $u \leqq p$ for some $p \in P$. Since $p \in[Q) \vee J$ there exist $q \in[Q)$ and $v \in J$ such that $p=q \wedge v$. Now $p \in(v] \cap K$ implies that $(v] \cap K \neq \varnothing$; therefore, by (2), there is a $w \in(v] \cap K$ such that $w \equiv 1(\Theta)$. Consequently $w \in Q$, implying that $p \in[Q)$, since $p \geqq q \wedge w$, and so $p \in Q$. This contradicts the disjointness of $P$ and $Q$ and so establishes our claim.

Consequently, again applying Stone's theorem, there is a prime ideal $P_{1}$ of $L$ such that $(P] \subseteq P_{1}$ and $P_{1} \cap([Q) \vee J)=\varnothing$. Now $P_{1}$ defines a congruence $\Phi$ on $L: u \equiv v(\Phi)$ if and only if both $u, v \in P_{1}$ or both $u, v \in L-P_{1}$. Since $x \in P_{1}$ and $y \in L-P_{1}$, we need only show that $\Theta \leqq \Phi$ to conclude that $x \not \equiv y(\Theta)$.

Clearly $J \subseteq[1] \Phi$ and thus $\Theta[J] \leqq \Phi$. Now let $a, b \in K, a \equiv b(\Theta)$. Since $[a] \Theta=[b] \Theta$, either both $a, b \in P \subseteq P_{1}$, or both $a, b \in Q \subseteq L-P_{1}$; that is, $a \equiv b(\Phi)$. We consequently conclude that

$$
\bar{\Theta}=\bigvee(\Theta(a, b) \mid a, b \in K, a \equiv b(\Theta)) \vee \Theta[J] \leqq \Phi .
$$

Thus we have shown that $x \not \equiv y(\Theta)$ implies $x \not \equiv y(\Theta)$, completing the proof of condition (ii) and concluding the proof of the lemma.

It should be noted that a corollary to Lemma 1 is the Congruence Extension Property for distributive lattices (not necessarily with 1 ). If $K, L$ is a pair of lattices, $K$ a sublattice of $L$, then we add a new greatest element 1 and let $L^{\prime}=L \cup\{1\}$, $K^{\prime}=K \cup\{1\}$. Applying Lemma 1 with $J=\{1\}$ yields the Congruence Extension Property.

Using our characterization of *-congruences we now establish the Congruence Extension Property for $\mathscr{B}_{\omega}$.

Theorem 1 (The Congruence Extension Property). Let $A$ and $B$ be pseudocomplemented distributive lattices and let $A$ be $a^{*}$-sublattice of $B$. Let $\Theta$ be a

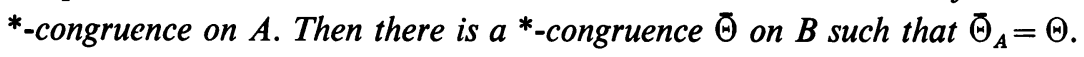

Proof. Let $\Theta_{1}=\Theta_{S(A)}, \Theta_{2}=\Theta_{D(A)}$. Note that $S(A)$ is a subalgebra of $S(B)$ and $D(A)$ is a $\{1\}$-sublattice of $D(B)$. Set $I_{1}=[1] \Theta_{1}$, and let $I$ be the dual ideal in $S(B)$ generated by $I_{1}$. In view of the one-to-one correspondence for Boolean algebras between dual ideals and congruences, $\left(\Theta_{1}\right)_{S(A)}=\Theta_{1}$, where $\Xi_{1}=\Theta[I]$.

Now let $J=\{x \in D(B) \mid x \geqq y$ for some $y \in I\}$. We establish condition (2) for the lattices $D(A), D(B)$ and the pair $J, \Theta_{2}$. Let $u \in J$ and let $w \in D(A), w \leqq u$. In view of the definitions of $J$ and $I$, there is an $a \in I_{1}$ such that $a \leqq u$. Since $a \in A$ and $w \in D(A)$ it follows that $a \vee w \in D(A) . a \in I_{1}$ implies that $a \equiv 1\left(\Theta_{1}\right)$ and so $a \vee w \equiv 1\left(\Theta_{2}\right)$. Thus condition (2) is established with $v=a \vee w$. Consequently there is a congruence $\Theta_{2}$ on $D(B)$ such that $\left(\Theta_{2}\right)_{D(A)}=\Theta_{2}$ and such that $x \equiv 1\left(\Theta_{2}\right)$ whenever $x \in J$. In view of the definition of $\Theta_{1}$, condition (1) thus applies to the pair $\left\langle\Theta_{1}, \Xi_{2}\right\rangle$; that is, $\left\langle\Theta_{1}, \widetilde{\Theta}_{2}\right\rangle$ is a congruence pair determining a *-congruence $\bar{\Theta}$ on $B$. Since $\left(\Theta_{1}\right)_{S(A)}=\Theta_{1}$ and $\left(\Theta_{2}\right)_{D(A)}=\Theta_{2}$ it follows that $\Theta_{A}=\Theta$, concluding the proof of the theorem. 
2. The standard semigroup. We apply Theorem 1 to calculate the standard semigroup of operators in the various equational classes of pseudocomplemented distributive lattices. Let $\mathscr{K}$ be an equational class of algebras. If $\mathscr{K}^{\prime} \subseteq \mathscr{K}$ let $\boldsymbol{H}\left(\mathscr{K}^{\prime}\right), \boldsymbol{S}\left(\mathscr{K}^{\prime}\right)$, and $\boldsymbol{P}\left(\mathscr{K}^{\prime}\right)$ denote the class of all homomorphic images, isomorphs of subalgebras, and isomorphs of direct products respectively of algebras in $\mathscr{K}^{\prime}$. With the set of symbols $\{\boldsymbol{H}, \boldsymbol{S}, \boldsymbol{P}\}$ we associate a partially ordered monoid $\subseteq(\mathscr{K})$. Let $\Sigma$ be the free monoid on $\{\boldsymbol{H}, \boldsymbol{S}, \boldsymbol{P}\}$ with the identity $\boldsymbol{0}$. If $\mathscr{K}^{\prime} \subseteq \mathscr{K}$ and $\boldsymbol{U}$ is a word in $\Sigma$ we define the class $U\left(\mathscr{K}^{\prime}\right)$ by requiring that $0\left(\mathscr{K}^{\prime}\right)=\mathscr{K}^{\prime}$ and $\boldsymbol{U} V\left(\mathscr{K}^{\prime}\right)$ $=\boldsymbol{U}\left(\boldsymbol{V}\left(\mathscr{K}^{\prime}\right)\right)$. Since $\mathscr{K}$ is an equational class $\boldsymbol{U}\left(\mathscr{K}^{\prime}\right) \subseteq \mathscr{K}$ for all $\mathscr{K}^{\prime} \subseteq \mathscr{K}, \boldsymbol{U} \in \Sigma$. The standard semigroup of operators of $\mathscr{K}, \Im(\mathscr{K})$, is the quotient monoid of $\Sigma$ where $\boldsymbol{U}, \boldsymbol{V} \in \Sigma$ are identified if $\boldsymbol{U}\left(\mathscr{K}^{\prime}\right)=\boldsymbol{V}\left(\mathscr{K}^{\prime}\right)$ for every subclass $\mathscr{K}^{\prime}$ of $\mathscr{K}$. The partial order is determined by setting $U \leqq V$ if $U\left(\mathscr{K}^{\prime}\right) \subseteq V\left(\mathscr{K}^{\prime}\right)$ for all $\mathscr{K}^{\prime} \subseteq \mathscr{K}$. Don Pigozzi [10] announced that, for each equational class $\mathscr{K}, S(\mathscr{K})$ is a quotient of the 18element partially order monoid $\subseteq$ depicted in Figure 1 , and showed the existence of a class $\mathscr{K}$ of groupoids for which $\mathfrak{S}(\mathscr{K})=\Im$.

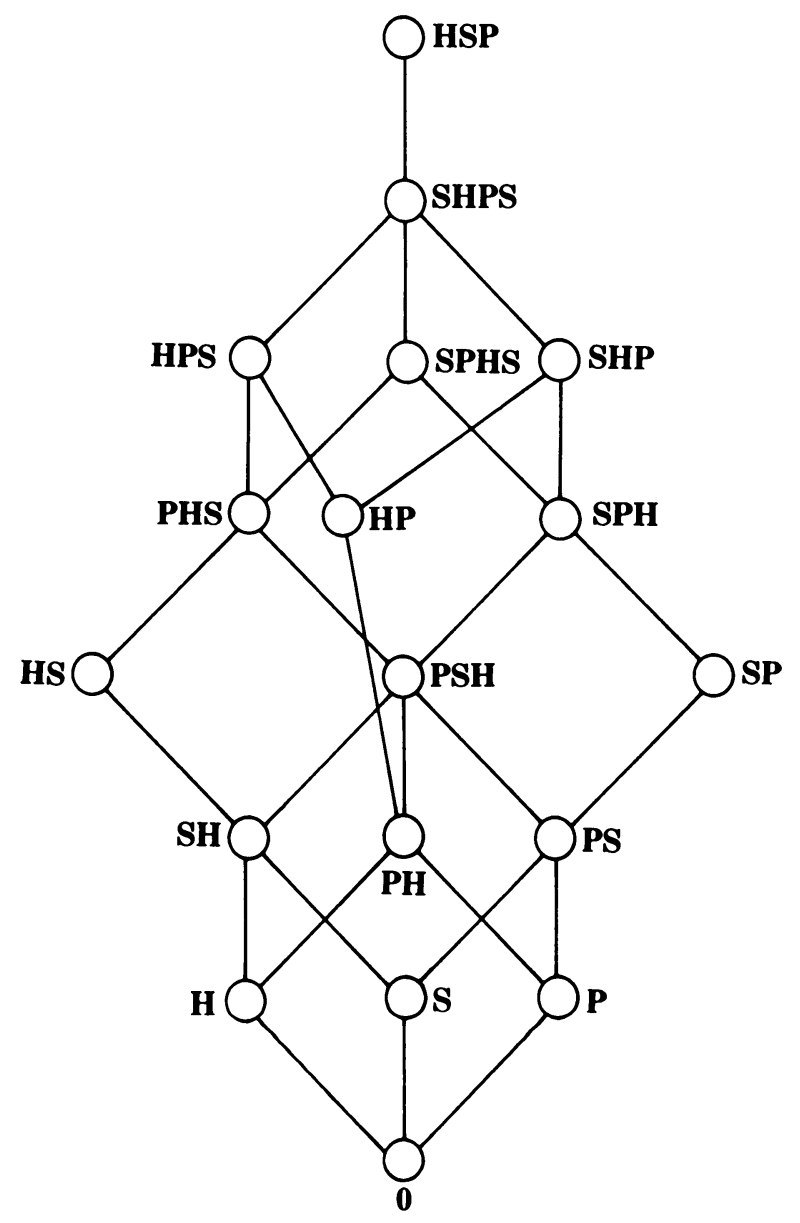

FIGURE 1 
Let $B$ be a Boolean algebra and let $\bar{B}$ be the pseudocomplemented distributive lattice obtained by adjoining a new greatest element to $B$. Then the $\bar{B}$ are exactly the subdirectly irreducible pseudocomplemented distributive lattices (Theorem 2 of Part I). Let $B_{n}$ denote the $n$-atom Boolean algebra and let $\mathscr{B}_{n}$ denote the equational class of pseudocomplemented distributive lattices determined by $\bar{B}_{n}$. Let $\mathscr{B}_{\omega}$ denote the class of all pseudocomplemented distributive lattices. It was shown by K. B. Lee [9] that the $\mathscr{B}_{n}, n$ finite, and $\mathscr{B}_{\omega}$ are all distinct and are all the nontrivial equational classes of pseudocomplemented distributive lattices (see also Part I). Observe that $\mathscr{B}_{0}$ is the class of all Boolean algebras.

THEOREM 2. (i) If $0 \leqq n \leqq 2$ then $\mathfrak{S}\left(\mathscr{B}_{n}\right)$ has 11 elements and is the quotient of $\mathfrak{S}$ under the relations $\boldsymbol{H S}=\boldsymbol{S H}, \boldsymbol{S P}=\boldsymbol{H S P}$.

(ii) If $2<n<\omega$ then $\mathfrak{S}\left(\mathscr{B}_{n}\right)$ has 12 elements and is the quotient of $\mathfrak{S}$ under the relations $\boldsymbol{H S}=\boldsymbol{S H}, \boldsymbol{S P H}=\boldsymbol{H S P}$.

(iii) $\mathfrak{S}\left(\mathscr{B}_{\omega}\right)$ has 13 elements and is the quotient of $\mathcal{S}$ under the relation $\boldsymbol{H S}=\boldsymbol{S H}$.

Proof. The Congruence Extension Property implies that $\boldsymbol{H S}=\boldsymbol{S H}$. The quotient of $\subseteq$ under this identification is the semigroup shown in Figure 2. Thus the standard semigroup of operators of any equational class of pseudocomplemented distributive

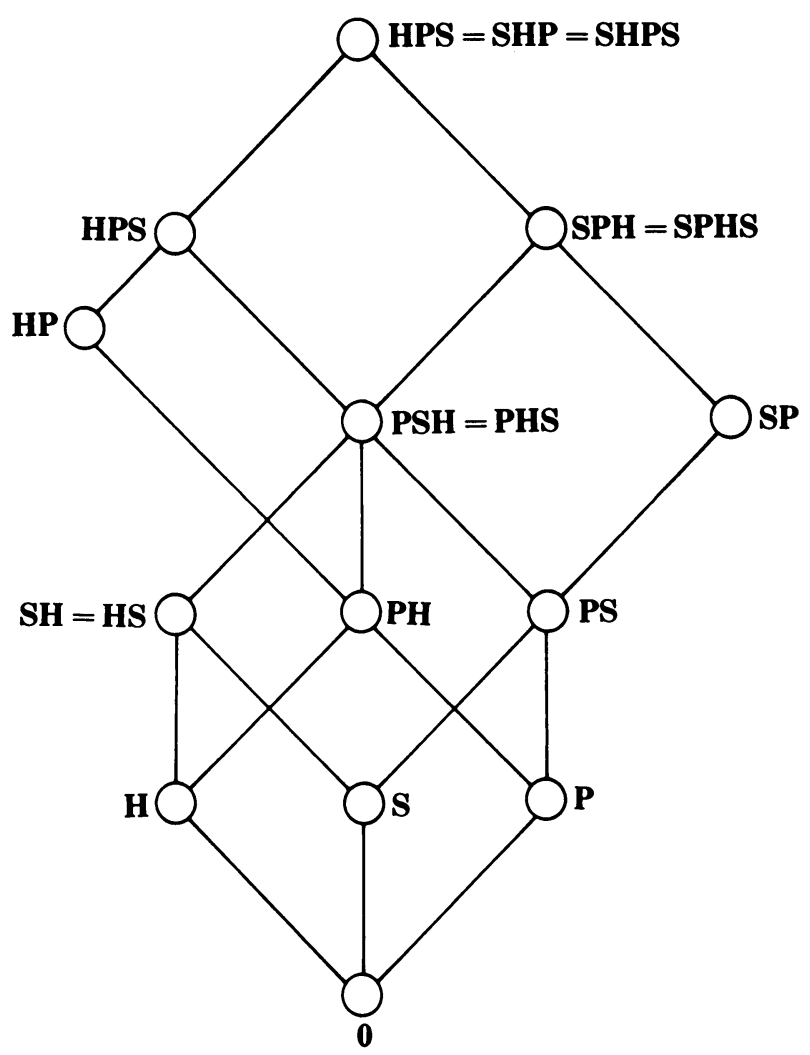

FIGURE 2 
lattices is a quotient of the semigroup of Figure 2 . Now $\mathscr{B}_{0}$ is a subclass of all nontrivial equational classes of pseudocomplemented distributive lattices. Consequently, $\mathfrak{S}\left(\mathscr{B}_{0}\right)$ is a quotient of each of the other semigroups. S. D. Comer and J. S. Johnson [3] state that $S\left(\mathscr{B}_{0}\right)$ is the quotient of $\subseteq$ under the identifications $\boldsymbol{H S}=\boldsymbol{S H}$ and $\boldsymbol{S P}=\boldsymbol{H S P}$, as given by Figure 3. A comparison of Figures 2 and 3

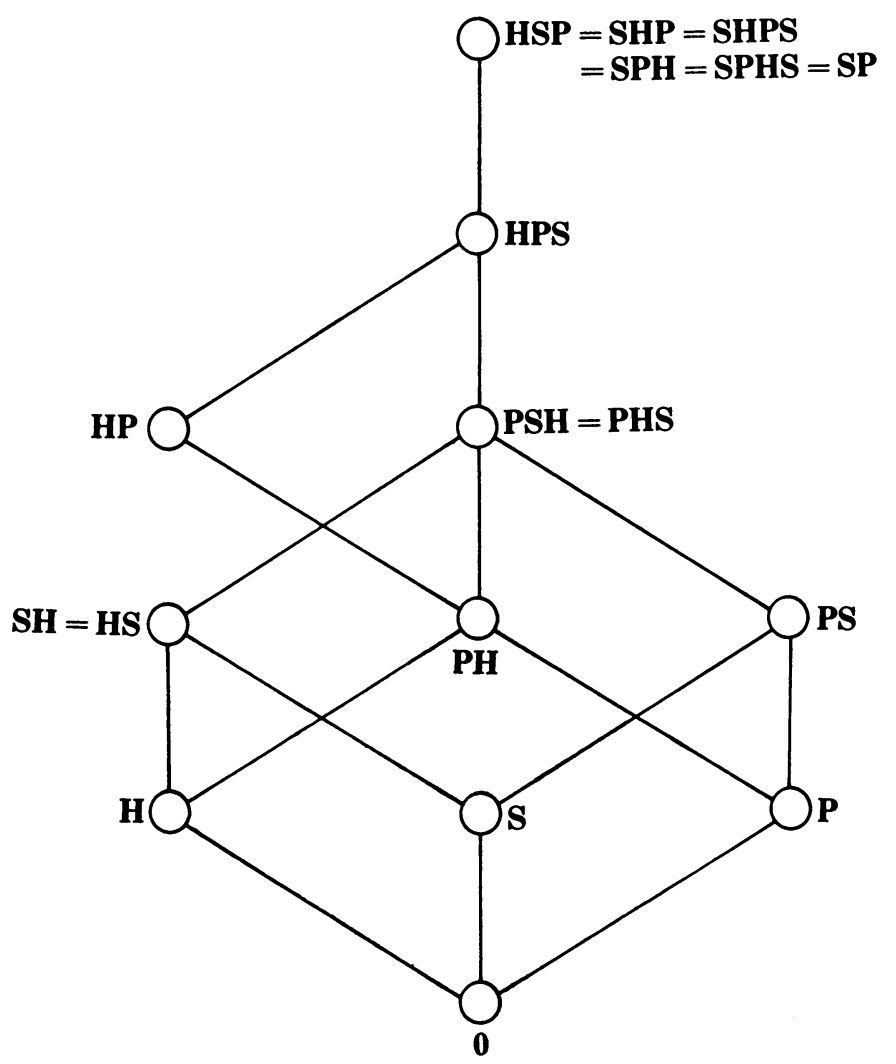

FIGURE 3

shows that for any nontrivial equational class of pseudocomplemented distributive lattices the set

$$
\{0, H, S, P, S H, P H, P S, H P, P S H, H P S\}
$$

consists of distinct elements and is disjoint with the set $\{\boldsymbol{S P}, \boldsymbol{S P H}, \boldsymbol{H S P}\}$. Observe further that $\boldsymbol{S P} \leqq \boldsymbol{S P H} \leqq \boldsymbol{H S P}$. It follows that the standard semigroup of operations of any nontrivial equational class of pseudocomplemented distributive lattices is a quotient of $\mathfrak{S}$ under one of the following four systems of defining relations:

(a) $\boldsymbol{H S}=\boldsymbol{S H}$;

(b) $\boldsymbol{H S}=\boldsymbol{S H}$ and $\boldsymbol{S P}=\boldsymbol{S P H}$;

(c) $H S=S H$ and $S P H=H S P$;

(d) $H S=S H$ and $S P=H S P$. 


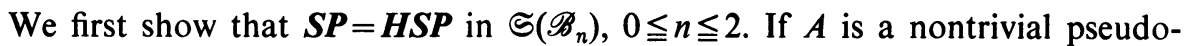
complemented distributive lattice then $\bar{B}_{0}=\{0,1\}$ is a subalgebra of $A$. Since $\boldsymbol{H S P}\left(\mathscr{K}^{\prime}\right)$ is the equational class generated by the class $\mathscr{K}^{\prime}$, we find that whenever $\mathscr{K}^{\prime} \subseteq \mathscr{B}_{0}$ is nontrivial then

$$
\boldsymbol{H S P}\left(\mathscr{K}^{\prime}\right)=\mathscr{B}_{0}=\operatorname{SP}\left(\bar{B}_{0}\right) \subseteq \boldsymbol{S P}\left(\mathscr{K}^{\prime}\right) \subseteq \boldsymbol{H S P}\left(\mathscr{K}^{\prime}\right)
$$

thus $\boldsymbol{H S P}=\boldsymbol{S P}$ in $\mathscr{B}_{0}$. If $\mathscr{K}^{\prime} \subseteq \mathscr{B}_{1}, \mathscr{K}^{\prime} \nsubseteq \mathscr{B}_{0}$, then there exist $A \in \mathscr{K}^{\prime}$ and a dense $u \in A, u \neq 1$. Since the subalgebra $\{0, u, 1\}$ of $A$ is isomorphic to $\bar{B}_{1}$ we conclude that

$$
\boldsymbol{H S P}\left(\mathscr{K}^{\prime}\right) \subseteq \mathscr{B}_{1}=\operatorname{SP}\left(\bar{B}_{1}\right) \subseteq \boldsymbol{S P}\left(\mathscr{K}^{\prime}\right) \subseteq \boldsymbol{H S P}\left(\mathscr{K}^{\prime}\right),
$$

establishing $\boldsymbol{H S P}=\boldsymbol{S P}$ for $\mathscr{B}_{1}$. If $\boldsymbol{A} \in \mathscr{B}_{2}-\mathscr{B}_{1}$, then, by the Subdirect Representation Theorem, there is a *-homomorphism of $A$ onto $\bar{B}_{2}$. Let $x \in A$ map onto one of the atoms of $\bar{B}_{2}$; then so does $x^{* *}$, and $x^{*}$ maps to the other atom. Thus the elements $0, x^{*}, x^{* *}, x^{*} \vee x^{* *}, 1$ of $A$ are all distinct and the set

$$
\left\{0, x^{*}, x^{* *}, x^{*} \vee x^{* *}, 1\right\}
$$

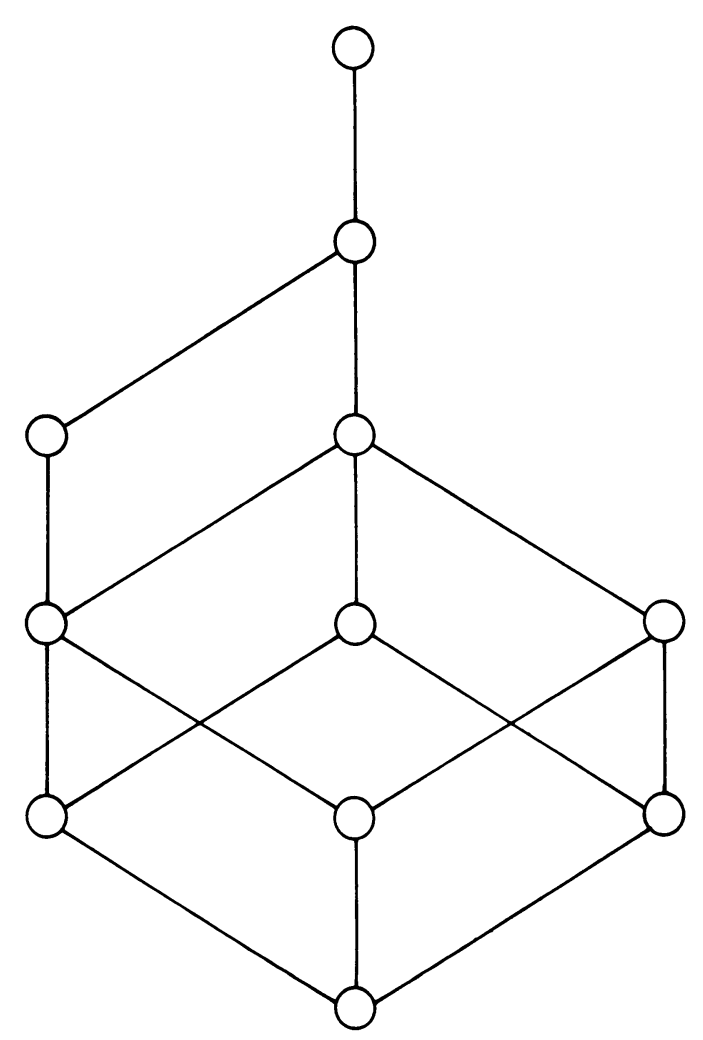

FIGURE 4 
is a subalgebra of $A$ isomorphic to $\bar{B}_{2}$. Thus, as above, $\boldsymbol{S P}\left(\mathscr{K}^{\prime}\right)=\boldsymbol{H} \boldsymbol{S P}\left(\mathscr{K}^{\prime}\right)$. We have thus established statement (i).

We now show that $\boldsymbol{S P H}=\boldsymbol{H S P}$ in $\left.\mathcal{S}_{(\mathscr{B}}\right)$ for $n<\omega$. Let $A \in \mathscr{B}_{n}-\mathscr{B}_{n-1}$; then $\bar{B}_{n}$ is a homomorphic image of $A$. Thus $\mathscr{K}^{\prime} \subseteq \mathscr{B}_{n}, \mathscr{K}^{\prime} \nsubseteq \mathscr{B}_{n-1}$ imply $\bar{B}_{n} \in \boldsymbol{H}\left(\mathscr{K}^{\prime}\right)$. Consequently

$$
\boldsymbol{H S P}\left(\mathscr{K}^{\prime}\right) \subseteq \boldsymbol{S P}\left(\bar{B}_{n}\right) \subseteq \operatorname{SPH}\left(\mathscr{K}^{\prime}\right) \subseteq \boldsymbol{H S P}\left(\mathscr{K}^{\prime}\right),
$$

establishing the identity $\boldsymbol{H S P}=\boldsymbol{S P H}$ for $\mathfrak{S}\left(\mathscr{B}_{n}\right)$.

Now let $2<n \leqq \omega$. Then $L \in \mathscr{B}_{n}$, where $L$ is a subdirect product of $\bar{B}_{2}$ and $\bar{B}_{3}$, and is depicted in Figure 4. Since $\bar{B}_{3}$ is a homomorphic image of $L, \boldsymbol{H} \boldsymbol{S P}(L)=\mathscr{B}_{3}$. We show that $\bar{B}_{3} \notin \boldsymbol{S P}(L)$. Assume, to the contrary, that $A$ is a direct power of $L$ and that $\bar{B}_{3}$ is isomorphic to a subalgebra of $A$. Since $\bar{B}_{3}$ is subdirectly irreducible, it would follow that $\bar{B}_{3}$ is isomorphic to a subalgebra of $L$ itself. That $L$ cannot contain an isomorph of $\bar{B}_{3}$ as a subalgebra is clear, since the three dual atoms in $S(L)$ do not all have the same pairwise join in $L$. Thus $\boldsymbol{S P} \neq \boldsymbol{S P H}$ in $\left.\mathfrak{S}_{(\mathscr{B}}\right), n>2$, concluding the proof of statement (ii).

To establish statement (iii) we need only show that $\boldsymbol{S P H} \neq \boldsymbol{H S P}$ in $\mathfrak{S}\left(\mathscr{B}_{\omega}\right)$. Let $B$ be an infinite Boolean algebra. If $A \in \boldsymbol{H}(\bar{B})$ then $A$ is either Boolean or isomorphic

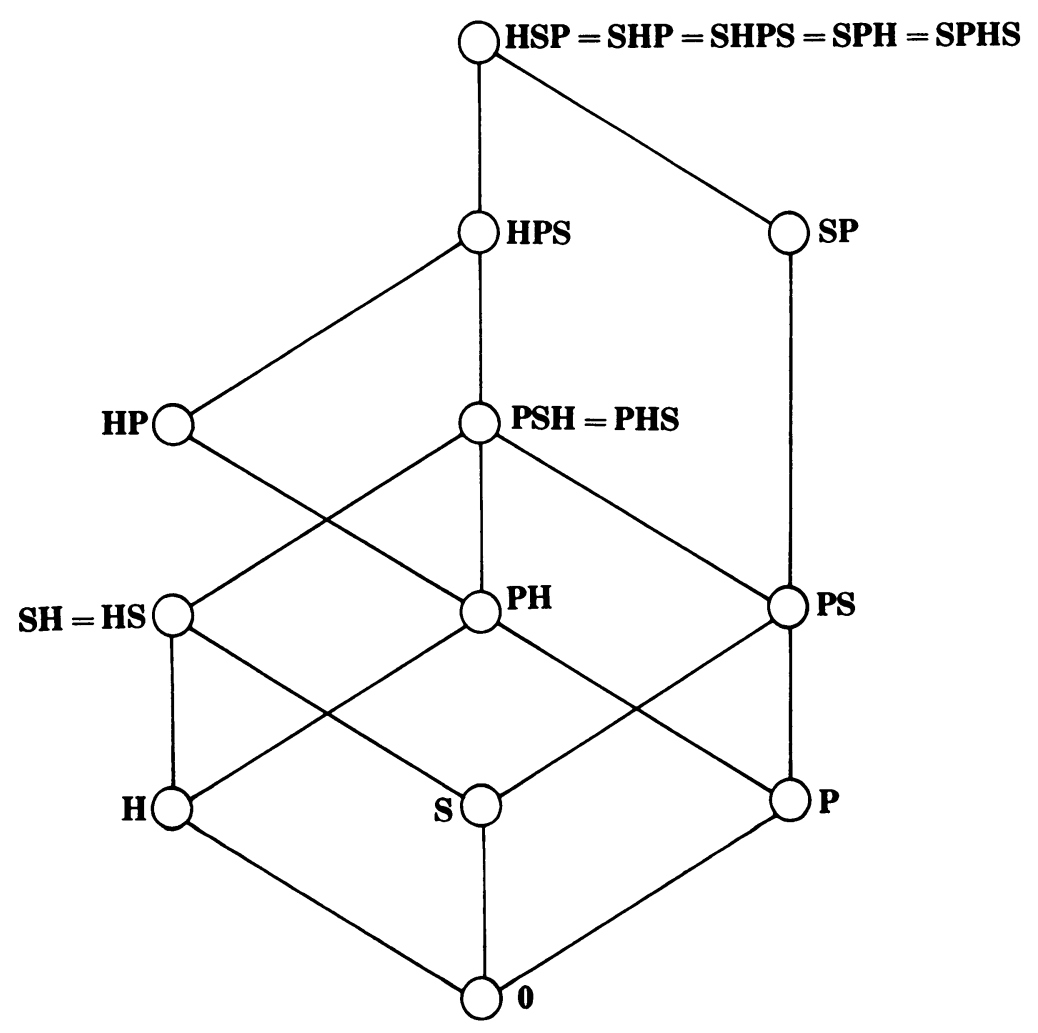

Figure 5 
to $\bar{B}$. On the other hand, $\boldsymbol{H S P}(\bar{B})=\mathscr{B}_{\omega}$. Consequently, if $B_{1}$ is a Boolean algebra of cardinality greater than that of $B$, then $\bar{B}_{1} \in \boldsymbol{H} \boldsymbol{S P}(\bar{B})-\boldsymbol{S P H}(\bar{B})$ since $\bar{B}_{1}$ is subdirectly irreducible. Thus statement (iii) is established, concluding the proof of the theorem.

$\mathfrak{S}\left(\mathscr{B}_{n}\right), 0 \leqq n \leqq 2$, is given in Figure 3, $S\left(\mathscr{B}_{n}\right), 2<n<\omega$, is depicted in Figure 5, and $\mathfrak{S}\left(\mathscr{B}_{\omega}\right)$ is shown in Figure 2.

3. The Amalgamation Property. Let $\mathscr{K}$ be a class of algebras. An amalgam in $\mathscr{K}$ is a triple $\langle A ; B, C\rangle$ of algebras in $\mathscr{K}$ such that $A$ is a common subalgebra of $B$ and $C$. The amalgam $\langle A ; B, C\rangle$ can be amalgamated in $\mathscr{K}$ if there is an algebra $D \in \mathscr{K}$ containing (up to isomorphism) $B$ and $C$ as subalgebras such that $A \subseteq B \cap C$. An equivalent definition is depicted in Figure 6.

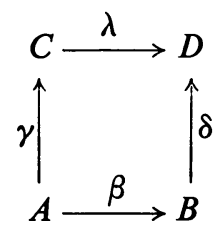

FIGURE 6

Given one-to-one homomorphisms $\beta: A \rightarrow B, \gamma: A \rightarrow C$ there are an algebra $D \in \mathscr{K}$ and one-to-one homomorphisms $\delta: B \rightarrow D, \lambda: C \rightarrow D$ such that the diagram commutes, that is, such that $\beta \delta=\gamma \lambda$. It is occasionally preferable to adopt this latter point of view, especially when $B=C$ and $\beta, \gamma$ are different embeddings of $A$. If this point of view is adopted we shall also denote the amalgam by $\langle A ; \beta, B, \gamma, C\rangle$. A class $\mathscr{K}$ of algebras is said to satisfy the Amalgamation Property if each amalgam in $\mathscr{K}$ can be amalgamated in $\mathscr{K}$. The development and importance of the Amalgamation Property in various branches of algebra are described in B. Jónsson [6].

In this section we determine which equational classes of pseudocomplemented distributive lattices satisfy the Amalgamation Property. We first present a simple condition equivalent to amalgamation in equational classes.

LEMmA 2. Let $\mathscr{K}$ be an equational class of algebras. The amalgam $\left\langle A ; B_{1}, B_{2}\right\rangle$ in $\mathscr{K}$ can be amalgamated in $\mathscr{K}$ if and only if the following condition holds:

(*) Given $i \in\{1,2\}, a_{1}, b_{1} \in B_{i}$ with $a_{1} \neq b_{1}$, there exist an algebra $D_{i} \in \mathscr{K}$ and homomorphisms $\varphi_{i}: B_{i} \rightarrow D_{i}, \psi_{i}: B, \rightarrow D_{i}$ such that $\varphi_{i}\left|A=\psi_{i}\right| A$ and $a_{1} \varphi_{i} \neq b_{1} \varphi_{i}$, where $i \neq j$ and $j \in\{1,2\}$.

Proof. If the amalgam $\left\langle A ; B_{1}, B_{2}\right\rangle$ can be amalgamated the conditions clearly hold. To prove the converse we need only let $D$ be the direct product of the various algebras $D_{1}$ and $D_{2}$ determined by all distinct pairs of elements from $B_{1}$ and from $B_{2}$. Then the family of the various homomorphisms $\varphi_{1}, \psi_{2}$ provides the embedding of $B_{1}$ in $D$, and the various homomorphisms $\psi_{1}, \varphi_{2}$ provide the embedding of $B_{2}$ in $D$, proving the lemma. 
To proceed further we need a universal algebraic lemma. Let $B$ be an algebra and let $A$ be a subalgebra of $B$. We say that $B$ is an essential extension of $A$ if any congruence on $B$ whose restriction to $A$ is trivial is itself trivial.

LEMMA 3. (a) An essential extension of a subdirectly irreducible algebra is itself subdirectly irreducible.

(b) Let $A$ be a subalgebra of an algebra $B$, and let $\Theta$ be a congruence on $B$. Then there is a congruence $\Phi$ on $B$ such that $\Theta \leqq \Phi, \Phi_{A}=\Theta_{A}$, and the extension $B / \Phi$ of $A / \Theta_{A}$ is essential.

Proof. To prove (a), let $A$ be subdirectly irreducible with smallest nontrivial congruence $\Theta_{0}$. Then there are $a, b \in A, a \neq b$, such that $a \equiv b\left(\Theta_{0}\right)$; thus $\Theta_{0}$ $=\Theta_{A}(a, b)$, the smallest congruence of $A$ identifying $a$ and $b$. Let $B$ be an essential extension of $A$ and let $\Phi$ be a nontrivial congruence on $B$. Then $\Phi_{A}$ is nontrivial implying that $a \equiv b(\Phi)$; consequently $\Theta_{B}(a, b)$ is the smallest nontrivial congruence on $B$, proving that $B$ is subdirectly irreducible.

To prove part (b), we note that, by Zorn's lemma, there is a congruence $\Phi$ on $B$ maximal with respect to the property that $\Theta \leqq \Phi$ and $\Phi_{A}=\Theta_{A}$. Since nontrivial congruences on $B / \Phi$ correspond to those congruences on $B$ that properly contain $\Phi$, it follows that the extension $B / \Phi$ of $A / \Theta$ is essential, concluding the proof of the lemma.

We obtain the following corollary to part (b).

COROllaRY. Let $\mathscr{K}$ be a class of algebras satisfying the Congruence Extension Property. Let $A, B \in \mathscr{K}$, let $A$ be a subalgebra of $B$, and let $\Theta$ be a congruence on $A$. Then there is a congruence $\Theta$ on $B$ such that $\bar{\Theta}_{A}=\Theta$ and such that the extension $B / \Theta$ of $A / \Theta$ is essential.

Part (a) of Lemma 3 can be found in R. A. Day [4]. Part (b) is essentially Proposition 2.5 , p. 258, of [2].

THEOREM 3. Let $\mathscr{K}$ be an equational class of algebras satisfying the Congruence Extension Property, and let every subalgebra of each subdirectly irreducible algebra in $\mathscr{K}$ be subdirectly irreducible. Then $\mathscr{K}$ satisfies the Amalgamation Property if and only if whenever $A, B, C$ are subdirectly irreducible algebras in $\mathscr{K}$ with $A$ a common subalgebra of $B$ and $C$, the amalgam $\langle A ; B, C\rangle$ can be amalgamated in $\mathscr{K}$.

Proof. The condition is clearly necessary for the Amalgamation Property to hold in $\mathscr{K}$. To prove the sufficiency, let $\langle A ; B, C\rangle$ be an amalgam in $\mathscr{K}$ and let $a, b \in B, a \neq b$. Since $B$ is a subdirect product of subdirectly irreducible algebras in $\mathscr{K}$, there is a subdirectly irreducible algebra $S_{1} \in \mathscr{K}$ and a homomorphism $\bar{\varphi}$ of $B$ onto $S_{1}$ such that $a \bar{\varphi} \neq b \bar{\varphi}$. By the hypotheses of the theorem, the algebra $A \bar{\varphi}$ is subdirectly irreducible. Applying Lemma 3(a) and the corollary to Lemma 3, we find a subdirectly irreducible extension $S_{2}$ of $A \bar{\varphi}$ and a homomorphism $\bar{\psi}$ from $B$ onto $S_{2}$ such that $\bar{\psi}|A=\bar{\varphi}| A$. Let the algebra $D$ in $\mathscr{K}$ amalgamate the amalgam 
$\left\langle A \bar{\varphi} ; S_{1}, S_{2}\right\rangle$, and let $\varphi: B \rightarrow D, \psi: C \rightarrow D$ respectively be the compositions of $\bar{\varphi}$ and $\bar{\psi}$ with the respective embeddings of $S_{1}$ and $S_{2}$ in $D$. Then, by symmetry, condition (*) of Lemma 2 holds, showing that $\langle A ; B, C\rangle$ can be amalgamated and concluding the proof of the theorem.

An interesting application of the Amalgamation Property is presented in B. Jónsson [7]. A pair of algebras $A, B$ can have a free product only if the constant subalgebra of $A$ is isomorphic to the constant subalgebra of $B$ or if neither $A$ nor $B$ have any constants $\left({ }^{2}\right)$. For the sake of convenience we shall describe either of these situations by saying that $A$ and $B$ have isomorphic constant subalgebras. An equational class $\mathscr{K}$ of algebras is said to admit free products if any two algebras in $\mathscr{K}$ with isomorphic constant subalgebras have a free product in $\mathscr{K}$. We denote the free product of $A$ and $B$ by $A * B$. An equational class $\mathscr{K}$ that admits free products is said to satisfy Property (P) if the following condition holds:

(P) Given algebras $A, B \in \mathscr{K}$ with isomorphic constant subalgebras, and given subalgebras $A_{1}, B_{1}$ of $A, B$ respectively, then the subalgebra of $A * B$ generated by $A_{1}$ and $B_{1}$ is (isomorphic to) $A_{1} * B_{1}$.

LEMMA 4. Let $\mathscr{K}$ be an equational class admitting free products. Then $\mathscr{K}$ satisfies Property (P) if and only if each amalgam $\langle A ; \beta, B, \alpha, A * C\rangle$ can be amalgamated in $\mathscr{K}$, where $A$ and $C$ are algebras in $\mathscr{K}$ with isomorphic constant subalgebras, $\beta: A \rightarrow B$ is an embedding with $B \in \mathscr{K}$, and $\alpha: A \rightarrow A * C$ is the canonical embedding.

Proof. Since $A$ is isomorphic to a subalgebra of $B$ it follows that $B * C$ exists. If Property (P) holds in $\mathscr{K}$ then $B * C$ is the amalgamation of the amalgam $\langle A ; \beta, B, \alpha, A * C\rangle$.

On the other hand, in order to prove Property (P) it suffices to show that if $B, C$ are algebras in $\mathscr{K}$ with isomorphic constant subalgebras and $A$ is a subalgebra of $B$ then the subalgebra $E$ of $B * C$ generated by $A$ and $C$ is $A * C$. Let $\beta: A \rightarrow B$ be the embedding. Let the algebra $D \in \mathscr{K}$ amalgamate $\langle A ; \beta, B, \alpha, A * C\rangle$, that is, there are embeddings $\delta: B \rightarrow D, \varepsilon: A * C \rightarrow D$ such that $\beta \delta=\alpha \varepsilon$. Let $\gamma: C \rightarrow A * C$ be the canonical embedding. Then the embeddings $\delta: B \rightarrow D$, $\gamma \varepsilon: C \rightarrow D$ yield a homomorphism $\varphi: B * C \rightarrow D$. If $x \in A \subseteq B * C$ then $x \varphi=x \beta \delta$ $=x \alpha \varepsilon$, and if $x \in C \subseteq B * C$ then $x \varphi=x \gamma \varepsilon$. Since $E$ is generated by $A$ and $C$ there is a homomorphism $\bar{\varphi}: E \rightarrow A * C$ such that $\bar{\varphi} \varepsilon=\varphi \mid E$. Since $\varepsilon$ is one-to-one, $\bar{\varphi} \mid A=\alpha$ and $\bar{\varphi} \mid C=\gamma$. Thus $\bar{\varphi}$ yields the required isomorphism between $E$ and $A * C$, concluding the proof.

An immediate corollary of Lemma 4 is that the Amalgamation Property implies Property (P), a result proved by B. Jónsson [7]. Our proof of Lemma 4 essentially follows Jónsson's approach, but is somewhat simpler.

There are examples of equational classes that satisfy Property (P) but do not satisfy the Amalgamation Property. For example, it is quite easy to show that the

${ }^{(2)}$ G. Grätzer [5] can be considered to be a general reference for the concepts from universal algebra used in this paper. 
class of all semigroups satisfies Property (P), but an example of N. Kimura (see $[1$, p. 139]) shows that the class of all semigroups does not satisfy the Amalgamation Property. However, if $\mathscr{K}$ has the Congruence Extension Property then the two properties are equivalent.

THEOREM 4. Let $\mathscr{K}$ be an equational class of algebras that admits free products and satisfies the Congruence Extension Property. Then $\mathscr{K}$ satisfies Property (P) if and only if it satisfies the Amalgamation Property.

Proof. That the Amalgamation Property implies Property (P) follows from Lemma 4, as observed above. Now let $\mathscr{K}$ satisfy Property (P) and let $\langle A ; \beta, B, \gamma, C\rangle$ be an amalgam in $\mathscr{K}$. Since $A$ is a subalgebra of $C, A$ and $C$ have isomorphic constant subalgebras and $A * C$ consequently exists. Consider the amalgam $\langle A ; \beta, B, \alpha, A * C\rangle, \alpha$ the canonical embedding of $A$ in $A * C$. By Lemma 4 there is an algebra $D$ in $\mathscr{K}$ and there are embeddings $\delta: B \rightarrow D, \lambda: A * C \rightarrow D$. Let $\varphi: A * C \rightarrow C$ be determined by $\gamma: A \rightarrow C$ and the identity on $C$. Then $\varphi$ is onto and, by the Congruence Extension Property, there is an algebra $E \in \mathscr{K}$, an embed$\operatorname{ding} \rho: C \rightarrow E$, and a homomorphism $\bar{\varphi}: D \rightarrow E$ such that $\lambda \bar{\varphi}=\varphi \rho$. (The situation is depicted in Figure 7.) Now $\alpha \varphi=\gamma$. Thus we have an algebra $E \in \mathscr{K}$, an embedding

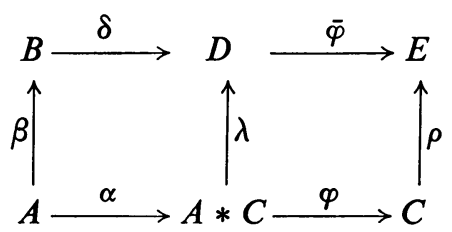

FIGURE 7

$\rho: C \rightarrow E$, and a homomorphism $\delta \bar{\varphi}: B \rightarrow E$ such that $\gamma \rho=\beta \delta \bar{\varphi}$. By Lemma 2 , and in view of the symmetry in the roles of $B$ and $C$, we conclude that $\langle A ; \beta, B, \gamma, C\rangle$ can be amalgamated in $\mathscr{K}$. Thus $\mathscr{K}$ satisfies the Amalgamation Property, and the proof is concluded.

We apply the above general results to pseudocomplemented distributive lattices.

THEOREM 5. Let $\mathscr{B}$ be a nontrivial equational class of pseudocomplemented distributive lattices. The following three conditions are equivalent:

(i) $\mathscr{B}$ satisfies the Amalgamation Property;

(ii) $\mathscr{B}$ satisfies Property (P);

(iii) $\mathscr{B}$ is one of $\mathscr{B}_{0}, \mathscr{B}_{1}, \mathscr{B}_{2}$, or $\mathscr{B}_{\omega}$.

Proof. The constant subalgebra of any nontrivial pseudocomplemented distributive lattice is isomorphic to $\bar{B}_{0}$. We observe later on in this proof that any amalgam of the form $\left\langle\bar{B}_{0} ; A, B\right\rangle$ can be amalgamated; thus any equational class of pseudocomplemented distributive latticès admits free products (see [5]). Since any equational class of pseudocomplemented distributive lattices satisfies the 
Congruence Extension Property, conditions (i) and (ii) are equivalent. We prove that conditions (i) and (iii) are equivalent.

Since each subdirectly irreducible pseudocomplemented distributive lattice is of the form $\bar{B}$ for some Boolean algebra $B$, it is immediately evident that a *-sublattice of a subdirectly irreducible pseudocomplemented distributive lattice is subdirectly irreducible. Consequently, by Theorem 3, we need only investigate amalgams of the form $\left\langle S_{0} ; S_{1}, S_{2}\right\rangle$ where $S_{0}, S_{1}$, and $S_{2}$ are subdirectly irreducible.

First consider the case where $S_{1}, S_{2}$ are both finite, say $S_{1}=\bar{B}_{m}, S_{2}=\bar{B}_{n}$ where $m \leqq n$. If $S_{0}$ is $\bar{B}_{0}$ or $\bar{B}_{1}$ there is only one embedding of $S_{0}$ into any $\bar{B}, B$ Boolean; map 0 to 0,1 to 1 , and, if $S_{0}=\bar{B}_{1}$, map the smaller of the two dense elements of $\bar{B}_{1}$ to the smaller of the two dense elements of $\bar{B}$. Consequently, if $S_{0}$ is $\bar{B}_{0}$ or $\bar{B}_{1}$, $\left\langle S_{0} ; \bar{B}_{m}, \bar{B}_{n}\right\rangle$ can be amalgamated by $\bar{B}_{n}$ since $\bar{B}_{m}$ is a ${ }^{*}$-sublattice of $\bar{B}_{n}$. We thus conclude that both $\mathscr{B}_{0}$ and $\mathscr{B}_{1}$ satisfy the Amalgamation Property.

We now consider $\mathscr{B}_{2}$. Let $\left\langle S_{0} ; S_{1}, S_{2}\right\rangle$ be an amalgam of subdirectly irreducible algebras in $\mathscr{B}_{2}$. If $S_{0}=\bar{B}_{0}$ or $\bar{B}_{1}$ then the above considerations show that $\left\langle S_{0} ; S_{1}, S_{2}\right\rangle$ can be amalgamated in $\mathscr{B}_{2}$. If $S_{0}=\bar{B}_{2}$ then $S_{0}=S_{1}=S_{2}$ since $\bar{B}_{2}$ is the greatest subdirectly irreducible algebra in $\mathscr{B}_{2}$. The amalgamation is thus trivial. Consequently, $\mathscr{B}_{2}$ satisfies the Amalgamation Property.

Since $\mathscr{B}_{0}$ is the equational class of Boolean algebras we obtain the well-known corollary that Boolean algebras satisfy the Amalgamation Property. We apply this result to prove the Amalgamation Property for $\mathscr{B}_{\omega}$. Let $A, B, C$ be Boolean algebras and let $\langle\bar{A} ; \bar{B}, \bar{C}\rangle$ be an amalgam of subdirectly irreducibles in $\mathscr{B}_{\omega}$. Then $\langle A ; B, C\rangle$ is an amalgam of Boolean algebras. If $\langle A ; B, C\rangle$ is amalgamated by the Boolean algebra $E$ then the pseudocomplemented distributive lattice $\bar{E}$ amalgamates $\langle\bar{A} ; \bar{B}, \bar{C}\rangle$. Thus $\mathscr{B}_{\omega}$ satisfies the Amalgamation Property.

Now consider $\mathscr{B}_{n}$ for $2<n<\omega$. Denote one of the atoms of $\bar{B}_{2}$ by $a$ and choose *-embeddings $\beta: \bar{B}_{2} \rightarrow \bar{B}_{n}, \gamma: \bar{B}_{2} \rightarrow \bar{B}_{n}$ such that $a \beta$ is an atom of $\bar{B}_{n}$ and $a \gamma$ is a dual atom of $B_{n}$. We show that the amalgam $\left\langle\bar{B}_{2} ; \beta, \bar{B}_{n}, \gamma, \bar{B}_{n}\right\rangle$ cannot be amalgamated in $\mathscr{B}_{n}$. Assume, to the contrary, that there is an algebra $C \in \mathscr{B}_{m}$ and that there are *-embeddings $\delta: \bar{B}_{n} \rightarrow C, \lambda: \bar{B}_{n} \rightarrow C$ such that $\gamma \lambda=\beta \delta$. By Lemma 3(b) there is an essential extension $D$ of $\bar{B}_{n}$ and a ${ }^{*}$-homomorphism $\rho$ of $C$ onto $D$ such that $\lambda \rho$ is the identity map on $\bar{B}_{n}$. However, an essential extension of $\bar{B}_{n}$ is subdirectly irreducible by Lemma $3($ a) and, since all subdirectly irreducible algebras in $\mathscr{B}_{n}$ are isomorphic to subalgebras of $\bar{B}_{n}$, it follows that $D=\bar{B}_{n}$; thus $\rho: C \rightarrow \bar{B}_{n}$; and $\rho$ maps $\bar{B}_{n}$ identically $\left({ }^{3}\right)$. Thus $\beta \delta \rho=\gamma$. Since $\gamma$ is one-to-one it does not collapse the two dense elements of $\bar{B}_{2}$. Consequently $\delta \rho$ does not collapse the two dense elements of $\bar{B}_{n}$. Since the smallest nontrivial congruence on $\bar{B}_{n}$ collapses the two dense elements of $\bar{B}_{n}$ we conclude that $\delta \rho: \bar{B}_{n} \rightarrow \bar{B}_{n}$ is one-to-one. Then $\delta \rho$ is an isomorphism, by the finiteness of $\bar{B}_{n}$. Thus $a \beta \delta \rho$ is an atom of $B_{n}$ and, since $a \gamma$ is a

$\left.{ }^{3}\right)$ What we have shown can be described as the fact that $\bar{B}_{n}$ is an absolute subretract in $\mathscr{B}_{n}$, a result we shall make use of in Part III of this series. 
dual atom of $B_{n}$ and $n>2$, we have found a contradiction to the requirement that $\gamma=\beta \delta \rho$. Thus $\left\langle\bar{B}_{2} ; \beta, \bar{B}_{n}, \gamma, \bar{B}_{n}\right\rangle$ cannot be amalgamated in $\mathscr{B}_{n}$, showing that $\mathscr{B}_{n}$ does not satisfy the Amalgamation Property if $2<n<\omega$. Thus we have established the equivalence of conditions (i) and (iii), concluding the proof of the theorem.

4. The amalgamation class. Let $\mathscr{K}$ be a class of algebras. We define the amalgamation class of $\mathscr{K}$, denoted Amal $(\mathscr{K})$ : an algebra $A \in \mathscr{K}$ belongs to Amal $(\mathscr{K})$ if and only if each amalgam $\langle A ; B, C\rangle$, where $B, C \in \mathscr{K}$, can be amalgamated in $\mathscr{K}$. Thus Amal $(\mathscr{K})=\mathscr{K}$ if and only if $\mathscr{K}$ satisfies the Amalgamation Property. In this section we present some results on Amal $\left(\mathscr{B}_{n}\right)$ for $2<n<\omega$. We first present a rather weak characterization of Amal $\left(\mathscr{B}_{n}\right)$.

Lemma 5. Let $n<\omega$ and let $A \in \mathscr{B}_{n}$. Then $A \in \operatorname{Amal}\left(\mathscr{B}_{n}\right)$ if and only if for any extension $C$ of $A$ in $\mathscr{B}_{n}$ and any ${ }^{*}$-homomorphism $\beta: A \rightarrow \bar{B}_{n}$ there is a *-homomorphism $\gamma: C \rightarrow \bar{B}_{n}$ extending $\beta$.

Proof. Let $A \in \operatorname{Amal}\left(\mathscr{B}_{n}\right)$ and let $\beta: A \rightarrow \bar{B}_{n}$. Define the *-embedding $\varphi: A \rightarrow A \times \bar{B}_{n}$ by the formula $a \varphi=\langle a, a \beta\rangle$. Let $C \in \mathscr{B}_{n}$ and let $\alpha: A \rightarrow C$ be a *-embedding. Since $A \in \operatorname{Amal}\left(\mathscr{B}_{n}\right)$ there are $D \in \mathscr{B}_{n}$ and *-embeddings $\delta: A \times \bar{B}_{n} \rightarrow D, \lambda: C \rightarrow D$ such that $\alpha \lambda=\varphi \delta$. Let $\eta: A \times \bar{B}_{n} \rightarrow \bar{B}_{n}$ be the projection onto the second factor. By the corollary to Lemma 3 there is a ${ }^{*}$-homomorphism $\rho: D \rightarrow \bar{B}_{n}$ such that $\delta \rho=\eta$. Then $\lambda \rho$ is the required extension $\gamma$ of $\varphi \eta=\beta$.

Now let $A$ satisfy the condition of the lemma. Let $\langle A ; B, C\rangle$ be an amalgam in $\mathscr{B}_{n}$, and let $a, b \in B, a \neq b$. Since $\mathscr{B}_{n}$ is generated by $\bar{B}_{n}$ there is a $*$-homomorphism $\bar{\beta}: B \rightarrow \bar{B}_{n}$ such that $a \bar{\beta} \neq b \bar{\beta}$. By our condition there is an extension $\gamma: C \rightarrow \bar{B}_{n}$ of $\bar{\beta} \mid A$. Applying Lemma 2 , we conclude that $A \in$ Amal $\left(\mathscr{B}_{n}\right)$, completing the proof.

We note that any *-homomorphism $\beta: A \rightarrow \bar{B}_{n}$ is the composition of a ${ }^{*}$-homomorphism of $A$ onto $\bar{B}_{i}$ for some $i \leqq n$ and a *-embedding of $\bar{B}_{i}$ into $\bar{B}_{n}$. Observing further that there is only one *-embedding of $\bar{B}_{i}$ into $\bar{B}_{n}$ if $i=0,1$, and applying the Congruence Extension Property, we get the following corollary.

Corollary. If $n<\omega, A \in \mathscr{B}_{n}$, and $A$ has no *-homomorphism onto $\bar{B}_{i}$ for any $i, 1<i<n$, then $A \in \operatorname{Amal}\left(\mathscr{B}_{n}\right)$.

If $A$ is finite we can determine whether or not $A \in \operatorname{Amal}\left(\mathscr{B}_{n}\right)$ in terms of the structure of $A$ alone.

THEOREM 6. Let $n<\omega$ and let $A$ be a finite pseudocomplemented distributive lattice in $\mathscr{B}_{n}$. Then $A \in \operatorname{Amal}\left(\mathscr{B}_{n}\right)$ if and only if $A$ has no *-homomorphism onto $\bar{B}_{i}$ for any $i, 1<i<n$.

Proof. If $A$ has no *-homomorphism onto $\bar{B}_{i}$ for any $i, 1<i<n$, then $A \in$ Amal $\left(\mathscr{B}_{n}\right)$ by the above corollary.

Now let $1<i<n$ and let $\varphi$ be a ${ }^{*}$-homomorphism of $A$ onto $\bar{B}_{i}$. We show that $A \notin$ Amal $\left(\mathscr{B}_{n}\right)$. Let the *-congruence $\Theta$ be the kernel of $\varphi$. By the Subdirect Decomposition Theorem and the finiteness of $A$ there are *-congruences $\Theta_{0}, \ldots, \Theta_{m}$ 
on $A$ such that $A / \Theta$, is subdirectly irreducible for all $j, 0 \leqq j \leqq m$, such that $\Theta_{0} \wedge \cdots \wedge \Theta_{m}=\omega$, the trivial congruence, and such that no proper subset of $\left\{\Theta_{0}, \ldots, \Theta_{m}\right\}$ meets in $\omega$. Since the lattice of *-congruences is distributive it follows that

$$
\Theta=\left(\Theta \vee \Theta_{0}\right) \wedge \cdots \wedge\left(\Theta \vee \Theta_{m}\right) .
$$

Since $A / \Theta$ is subdirectly irreducible there is a $j$ such that $\Theta=\Theta \vee \Theta_{j}$, that is, such that $\Theta_{j} \leqq \Theta$. It follows thus that $A / \Theta$ is a homomorphic image of the subdirectly irreducible $A / \Theta_{j}$. However the only proper homomorphic image of $A / \Theta_{j}$ is a Boolean algebra; consequently $\Theta=\Theta_{j}$. Set $\Phi=\Theta_{0} \wedge \cdots \wedge \Theta_{j-1} \wedge \Theta_{j+1} \wedge \cdots \wedge \Theta_{m}$. Then $\Phi \neq \omega$ and $\Theta \wedge \Phi=\omega$. Let $\rho: A \rightarrow A / \Phi$ be the canonical homomorphism. Since $1<i<n$ there is an atom $a \in \bar{B}_{i}$ and there are *-embeddings $\alpha, \beta: \bar{B}_{i} \rightarrow \bar{B}_{n}$ such that $a \alpha$ is an atom of $\bar{B}_{n}$ but $a \beta$ is not an atom of $\bar{B}_{n}$. Map $A$ to $A / \Phi \times \bar{B}_{n}$ by mapping $x$ to $\langle x \rho, x \varphi \alpha\rangle$; since $\Phi \wedge \Theta=\omega$ this mapping embeds $A$ in $A / \Phi \times \bar{B}_{n}$. Define $\gamma: A \rightarrow \bar{B}_{n}$ by the formula $x \gamma=x \varphi \beta$. We show that $\gamma$ has no extension to $A / \Phi \times \bar{B}_{n}$ and thus, by Lemma 5 , that $A \notin \operatorname{Amal}\left(\mathscr{B}_{n}\right)$.

Since the lattice of ${ }^{*}$-congruences of any pseudocomplemented distributive lattice is distributive, the lattice of *-congruences of $A / \Phi \times \bar{B}_{n}$ is isomorphic to the direct product of the lattices of *-congruences of $A / \Phi$ and $\bar{B}_{n}$. Thus if $\delta: A / \Phi \times \bar{B}_{n} \rightarrow \bar{B}_{n}$ is an extension of $\gamma: A \rightarrow \bar{B}_{n}$ we can represent the kernel of $\delta$ as $\left\langle\Theta_{0}, \Theta_{1}\right\rangle$ where $\Theta_{0}$ is a ${ }^{*}$-congruence on $A / \Phi$ and $\Theta_{1}$ is a ${ }^{*}$-congruence on $\bar{B}_{n}$. Since $\left(A / \Phi \times \bar{B}_{n}\right) \delta$ is a subalgebra of $\bar{B}_{n}$ it is subdirectly irreducible; thus $\left\langle\Theta_{0}, \Theta_{1}\right\rangle$ is meet irreducible. Thus either $\Theta_{0}=\imath$, the congruence collapsing all elements of the algebra, or $\Theta_{1}=\imath$. If $\Theta_{0}=\imath$ then $\langle y, z\rangle \delta=z$. Consequently $x \varphi \alpha=x \phi \beta$ for all $x \in A$, contradicting the definition of $\alpha$ and $\beta$. On the other hand, $\Theta_{1}=\iota$ implies that $\Phi \leqq \Theta$ since $\delta$ is an extension of $\gamma=\varphi \beta$ and $\beta$ is one-to-one. This conclusion contradicts the requirements that $\Phi \neq \omega$ and $\Phi \wedge \Theta=\omega$. We thus conclude that $\gamma$ can have no extension to $A / \Phi \times \bar{B}_{n}$, and so that $A \notin \operatorname{Amal}\left(\mathscr{B}_{n}\right)$ by Lemma 5 . The proof of the theorem is thus concluded.

In the proof of Theorem 6 the finiteness of $A$ is essential. It seems reasonable, though, that Theorem 6 holds for infinite algebras as well. We thus conclude this paper with the following problem.

Does Theorem 6 hold for infinite pseudocomplemented distributive lattices? If not, what is an intrinsic characterization of the algebras in Amal $\left(\mathscr{B}_{n}\right)$ for $2<n<\omega$ ?

\section{BIBLIOGRAPHY}

1. A. H. Clifford and G. B. Preston, The algebraic theory of semigroups. Vol. II, Math. Surveys, no. 7, Amer. Math. Soc., Providence, R. I., 1967. MR 36 \#1558.

2. P. M. Cohn, Universal algebra, Harper and Row, New York, 1965. MR 31 \#224.

3. S. D. Comer and J. S. Johnson, The standard semigroup of operators of a variety (manuscript).

4. R. A. Day, Injectives in non-distributive equational classes of lattices are trivial, Arch. Math. (to appear). 
5. G. Grätzer, Universal algebra, Van Nostrand, Princeton, N. J., 1968. MR 40 \#1320.

6. B. Jónsson, Extensions of relational structures, Proc. Internat. Sympos. Theory of Models (Berkeley, 1963), North-Holland, Amsterdam, 1965, pp. 146-157. MR 34 \#2463.

7. —_, Sublattices of a free lattice, Canad. J. Math. 13 (1961), 256-264. MR 23 \#A818.

8. H. Lakser, The structure of pseudocomplemented distributive lattices. I: Subdirect decomposition, Trans. Amer. Math. Soc. 156 (1971), 335-342.

9. K. B. Lee, Equational classes of distributive pseudo-complemented lattices, Canad. J. Math. 22 (1970), 881-891.

10. D. Pigozzi, On some operations on classes of algebras, Notices Amer. Math. Soc. 13 (1966), 829. Abstract \#639-1.

UNIVERSITY OF MANITOBA,

Winnipeg, Manitoba, Canada 